\title{
ÉTUDE DES CORRESPONDANCES ENTRE DEUX MÉTHODES D'APPRÉCIATION DE L'ÉTAT D'ENGRAISSEMENT DES CARCASSES DE BOVINS
}

\author{
B. L. DUMONT, G. ROY et J. LEFEBVRE* \\ Laboratoire de Recherches sur la Viande, \\ * Laboratoire de Génétique factorielle, \\ Centre national de Recherches zootechniques, I. N. R. A., \\ 78350 Jouy en Josas
}

\begin{abstract}
RÉSUMÉ
Par analyse factoriclle des correspondances et par analyse progressive de variance, les résultats du jugement subjectif de l'état d'engraissement des carcasses de bovins par une méthode descriptive ont été comparés aux mesures d'épaisseur de la graisse de couverture à onze niveaux de la carcasse. On a trouvé que les épaisseurs de graisse aux différents sites participaient inégalement à l'élaboration, dans l'esprit du juge, de la note d'appréciation. Il est proposé d'estimer l'état d'engraissement des carcasses à partir de l'épaisseur de gras à doux niveaux de la carcasse.
\end{abstract}

\section{INTRODUCTION}

Pour apprécicr rapidement l'état d'engraissement des carcasses de bovins on a proposé récemment (Roy et Dumont, 1975) une méthode de jugement subjectif reposant sur l'estimation visuelle de l'importance des graisses dans les différentes zones de la carcasse. Cette appréciation revêt maintenant une grande importance commerciale en raison de la prise en considération de l'état d'engraissement dans le classement obligatoire des carcasses (arrêté du I4 mai 1975. Journal Officiel de la République Française en date du 4 juin 1975,5576 -5577).

La généralisation de la pratique du classcment pose le problème de son contrôle ; il est souhaitable, à cet égard, que le jugement subjectif puisse être, le cas échéant, confronté et appuyé par les résultats de méthodes objectives. 
La présente note rapporte la comparaison des résultats obtenus en appliquant la méthode de Roy et Dumont (1975) et en appréciant, par ailleurs, l'épaisseur de la graisse sous-cutanée à différents niveaux de la carcasse.

\section{MA'TÉRIEL E’T MÉTHODES}

Le matériel animal et les méthodes de mesure de la graisse sous-cutanée ont été précédemment décrits (Dumont et Roy, 1975). Sur chaque carcasse on a procédé de façon indépendante au jugement de l'état d'engraissement selon la méthode de Roy et DUNoNi (i97.5) et à la mesure des épaisseurs de graisse en onze sites $(A, B \ldots K)$ défunis comme suit :

(A) Latéralement à la carcasse sur une horizontale passant au niveau du bord supérieur de l'apophyse épineuse de la quatrième vertèbre thoracique. Le point A est à mi-distance du plan médian du corps et de la limite inférieure de la portion dorsale du muscle Trapèze.

(B) Sur l'épine acromienne du Scapulum, au niveau de la tubérosité acromienne.

(C) Latéralement à la carcasse à $5 \mathrm{~cm}$ du plan médian du corps sur une horizontale passant au niveau du bord supéricur de l'apophyse épineuse de la septième vertèbre thoracique.

(D) Au même niveau que le point $\mathrm{C}$, mais à $\mathrm{Io} \mathrm{cm}$ de côté du plan médian du corps.

(E) Sur une horizontale passant par le milieu de l'apophyse épincuse de la première vertèbre lombaire, à $5 \mathrm{~cm}$ de côté du plan médian du corps.

(F) Sur une horizontale passant par le milieu de l'apophyse épincuse de la première vertèbre lombaire, à $5 \mathrm{~cm}$ de côté du plan médian du corps.

(G) Sur une horizontale passant par le milieu de l'apophyse épineuse de la quatrième vertèbre sacrée, à $5 \mathrm{~cm}$ de côté du plan médian du corps.

(H) Sur une horizontale passant par le milieu de l'apophyse épineuse de la quatrième vertèbre sacrée, à $\mathrm{I} 5 \mathrm{~cm}$ de côté du plan médian du corps.

(I) Dans le sillon séparant les muscles Somitendinosus et Semimembranosus, au niveau de 1'horizontale passant par le périnée.

(J) Sur le muscle Biceps femoris dans la partie médiane du sillon qui sépare les deux portions de ce inuscle.

(K) Au niveau du périnée, dans le plan de section de la carcasse, à l'aplomb de la verticale passant par le bord antéro-inféricur de la symphyse ischio-pubienne.

Le traitement statistique a été conduit en deux étapes successives :

- par l'analyse factorielle des correspondances (BENZECRT, 1964) appliquée à l'ensemble des données ;

- par l'étude des régressions multiples progressives avec le choix des variables d'épaisscurs de graisse explicatives de la note subjective de l'état d'engraissement.

\section{RÉSUL'TATS ET DISCUSSION}

Le poids moyen des carcasses était de $322.6-4 \cdot 69.5 \mathrm{~kg}$ et la note moyenne d'état d'engraissement était de $7,96: 2,6_{4}$.

Ainsi cléfini, l'échantillon d'animaux de cette étucle correspondait à un enscmble regroupant des types commerciaux très variés et manifestant touss les intermédiaires entre l'extrême maigreur et un engraissement très important.

Le tableau $\mathrm{I}$ indique la valeur des coefficients de corrélation entre les épaisscurs aux différents sites et le poids de la carcasse d'une part, et la note subjective d'état d'engraissement d'autre part. Il apparaît nettement que ni les épaisscurs de gras ni la note subjective ne sont liées au poids de la carcasse. Par contre il existe des liaisons positives et significatives $(P<0,0 r)$ entre les épaisseurs aux différents niveaux et la note subjective. Il est donc clair que l'accroissement des dépôts graisseux aux différents niveaux consiclérés dans cette étude participe, d'une manière ou d'une autre, à l'élaboration de l'impression d'état d'engraissement plus ou moins marqué que laisse, dans l'esprit du juge, l'examen visuel de la carcasse. 
APPRÉCIATION DÊ L'ÉTAT D'ENGRAISSEMENT

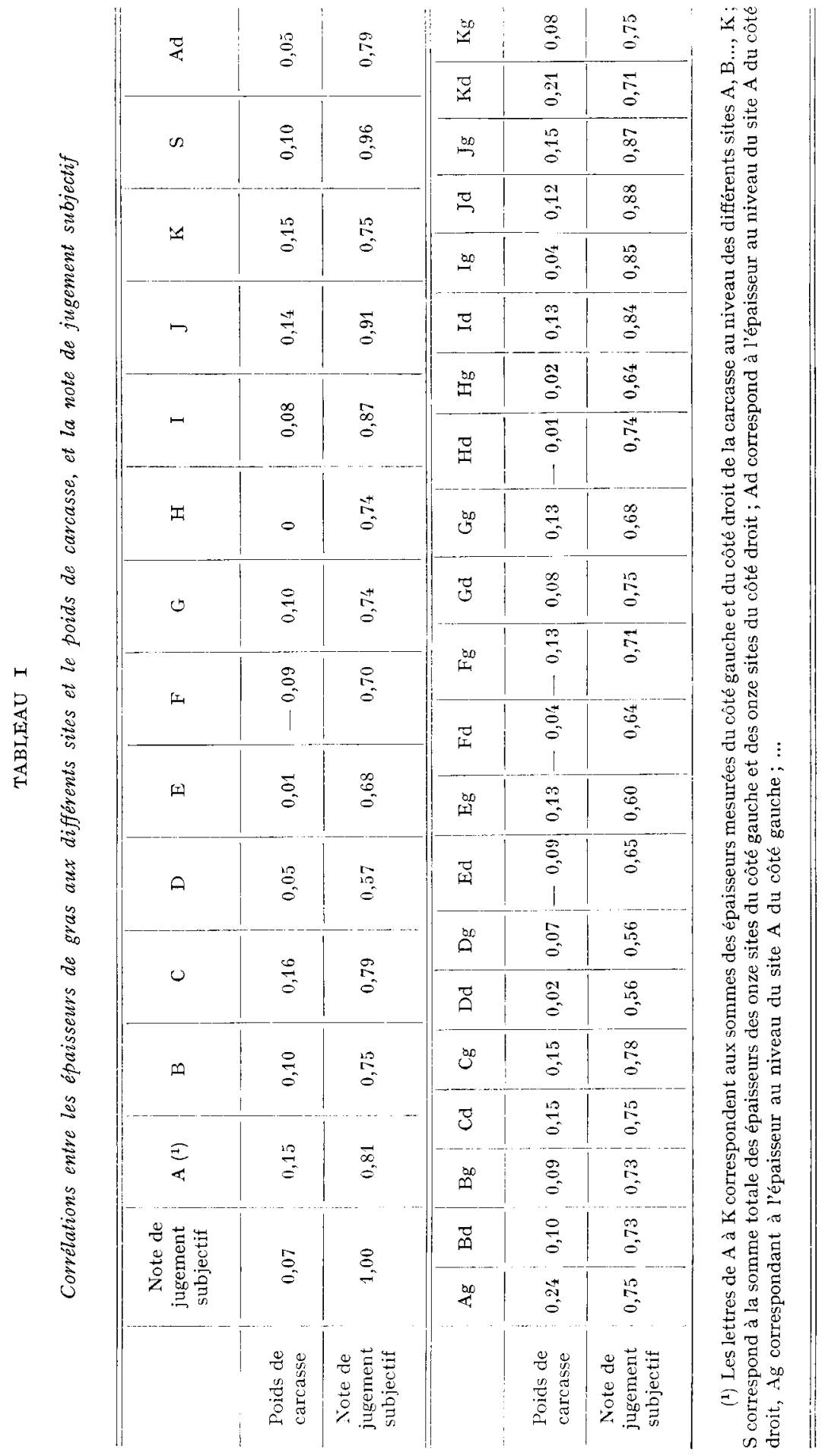


Les relations entre le poids de carcasse et les épaisseurs de gras aux différents niveaux sont illustrées, dans l'analyse des correspondances, par la position occupée par la projection de ces variables sur les différents axes factoriels (fig. I). Le poids de carcasse est en effet très proche du centre de gravité alors que les épaisseurs de gras forment un nuage qui en est nettement séparé.
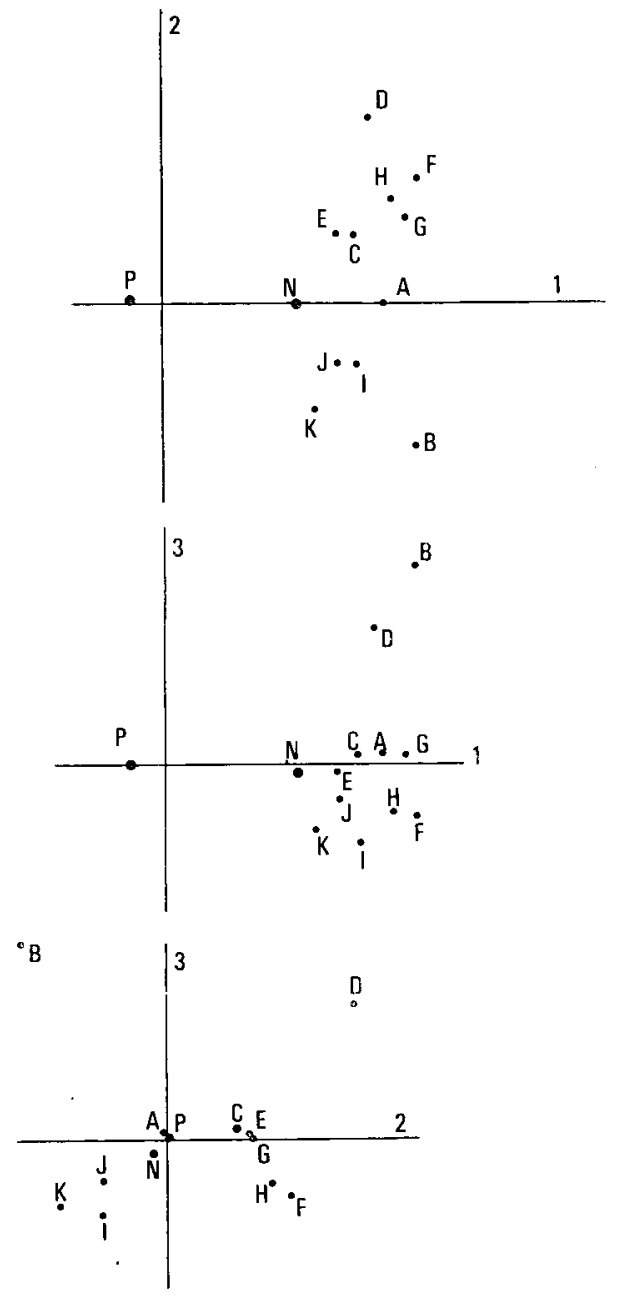

FIG. I. - Projection des variables sur les différents axes factoriels (A à K, mesures d'épaisseurs de gras;

$X$, note subjective d'état d'engraissement; $P$, poids de carcasse)

La projection sur les différents axes fait apparaitre aussi une nette séparation entre certains groupes d'épaisseurs, par exemple le groupe (C, E, F, G et H) et le groupe (I, J, K) sur le deuxième facteur. On observe aussi la très faible dispersion existant sur les axes successifs de projection des épaisseurs aux sites I, J et $\mathrm{K}$. On note enfin une variation importante, quels que soient les axes considérés, dans les distances qui séparent la note subjective des épaisseurs aux divers sites étudiés, les sites $\mathrm{C}$, E et J étant généralement les plus près cle la note subjective. Ces observations amènent à penser que si lá párticipation des épaisseurs de gras à l'élaboration de la note d'appréciation subjective est nette, elle intervient plus ou moins fortement selon les sites. 
Cette interprétation des résultats fournis par la méthode d'analyse factorielle est confirmée par l'analyse progressive de la variance. Les différents sites contribuent diversement à la formation de la note par le juge. Ce fait sera discuté en considérant comme variables d'épaisseurs les sommes des épaisseurs du côté gauche et du côté droit du même site anatomique. L'importance différente des contributions de chaque site est bien mise en évidence dans le tableau 2 où sont indiquées les valeurs de $\mathrm{R}^{2}$ dans le cas de régressions multiples lorsque les variables sont introduites dans l'ordre arbitraire de leur définition (A, B, C... J, K) et dans l'ordre de leur valeur explicative relative. On voit alors que les sites D, E, F, G, II n'apportent pas, par eux-mêmes, une fois considéré l'ensemble de tous les autres sites, un élément particulier d'explication de la notation du juge. On remarquera que les cinq zones que nous considérons comme moins intéressantes pour expliquer les variations de jugement de l'expert se trouvent localisées sur la face externe de la carcasse, dans la région dorso-lombaire supérieure, zone où la couverture graisseuse est d'apparence uniforme dans son étendue et où les variations d'épaisseurs, dans un site donné, ne s'accompagnent pas d'une progression périphérique marquée de la graisse en surface comme c'est le cas pour la graisse qui recouvre la cuisse ou la région du membre antérieur.

\section{TABLEAU 2}

$V$ aleur des $R^{2}$ en fonction du nombre

et de la nature des variables explicatives introduites successivement dans les équations de régression multiple d'estimation de la note de jugement subjectif

\begin{tabular}{c|c|c|c|c|c|c|c|c|c|c|c}
\hline & $\mathrm{A}$ & $\mathrm{B}$ & $\mathrm{C}$ & $\mathrm{D}$ & $\mathrm{E}$ & $\mathrm{F}$ & $\mathrm{G}$ & $\mathrm{H}$ & $\mathrm{I}$ & $\mathrm{J}$ & $\mathrm{K}$ \\
\hline $\begin{array}{c}\text { Introduction } \\
\text { arbitraire }\end{array}$ & $\mathrm{0,663}$ & 0,752 & 0,805 & 0,805 & 0,825 & 0,839 & 0,850 & 0,860 & 0,922 & 0,930 & 0,935 \\
\hline \hline & $\mathrm{C}$ & $\mathrm{J}$ & $\mathrm{I}$ & $\mathrm{A}$ & $\mathrm{I}$ & $\mathrm{K}$ & & & \\
\hline $\begin{array}{c}\text { Analyse } \\
\text { progressive }\end{array}$ & 0,629 & 0,901 & 0,910 & 0,916 & 0,921 & 0,922 & & \\
\hline
\end{tabular}

A partir des résultats de cette étude, on peut tenter d'apprécier l'objectivité, sinon la réalité du jugement subjectif réalisé à partir de l'examen descriptif de la carcasse. Au vu des valeurs des corrélations multiples obtenues on est fondé à considérer comme satisfaisantes la méthode subjective proposée et l'application qui en a été faite. La part de la variation résiduelle de la note subjective non expliquée par les mesures d'épaisseur de gras est faible et peut être imputée au fait que le jugement subjectif considère l'état d'engraissement de façon globale en tenant compte également (pour 20 p. Ioo) dans la fixation de la note de l'ensemble des graisses internes tapissant les cavités thoracique et abdominale.

On peut suggérer, à partir de nos résultats, que la mesure de l'épaisseur de graisse à différents niveaux, outre le fait qu'elle permet d'établir un profil utile pour caractériser le type de carcasse (Dumont et Roy, 1975), pourrait servir à contrôler le jugement subjectif de l'état d'engraissement en cas de besoin (litiges par exemple) dans la pratique courante de son exécution.

L'estimation de la note $(Y)$ peut se faire à partir de la mesure des épaisseurs (en centimètres) aux sites $\mathrm{C}\left(x_{c}\right)$ et $\mathrm{J}\left(x_{j}\right)$ d'après l'équation suivante :

$$
\text { (I) } \quad \mathrm{Y}=2,25+\mathrm{I}, 50 x_{c}+\mathrm{I}, 45 x_{j} \quad \begin{aligned}
& s_{y}= \pm 0,84 \\
& \mathrm{R}^{2}=0,90 \mathrm{I}
\end{aligned}
$$


La figure 2 indique la répartition, par classes de o,5 point, des écarts entre la valeur réelle et la valeur estimée à partir de l'équation ( $\mathbf{I}$ ) de la note d'état d'engraissement des différentes carcasses considérées dans ce travail. L'écart le plus important entre deux valeurs a été de 2,06 point.

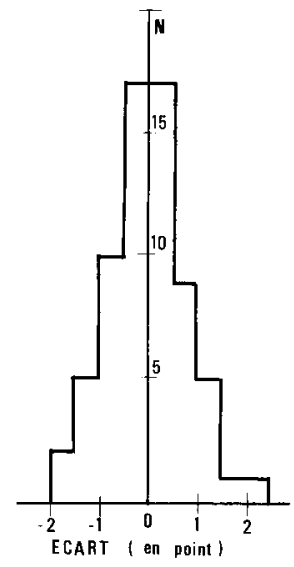

FIG. 2. - Répartition, par classes de 0,5 point,

des écarts entre la valeur réelle attribuée par l'expert

et la valeur estimée de la note d'état d'engraissement à partir de l'équation ( $\mathbf{r}$ )

D'autre part la comparaison entre les notes de jugement subjectif et les mesures d'épaisseurs pourrait servir à déceler d'éventuelles modifications de la couche de graisse de couverture provoquées par des ablations délibérées de ce tissu au cours des opérations de préparation des carcasses.

Reçu pour publication en juin 1975.

\title{
SUMMARY
}

\author{
RELATIONSHIPS BETWEEN TWO METHODS \\ OF JUDGING FATNESS OF CATTLE CARCASSES
}

Measurements of subcutaneous fat at elcven levels of the carcass have been compared to the scores given by a judge using a subjcctive method for assessing fatness.

It was shown by multidimensional analysis that fat thickness at the different levels were not associated in the same way in the judge's mind with his own impression on the overall fatness of the carcass. His scoring was highly correlated $\left(\mathrm{R}^{2}=0,90 \mathrm{I}\right)$ with the fat thickness at two levels : the first on the back $-5 \mathrm{~cm}$ laterally to the backbone, at the level of the 7 th thoracic vertebra, and the second on the thigh, in the middle part of the Biceps femoris muscle.

\section{RÉFÉRENCES BIBLIOGRAPHIQUES}

Benzecri J. P., I964. Cours de linguistique mathématique. Faculté des Sciences de Rennes.

J) umont B. L., Roy G., I975. Note sur la variation topographique de l'épaisseur de la graisse de coulverture chez les bovins. Ann. Zootech., 24, 587-593.

Roy G., Dumont B. L., I975. Méthode de jugement descriptif de l'état d'engraissement des carcasses de bovins. Rev. Méd. vet., 126, 3, 387-4oo. 